\title{
Adaptive Neural Networks Control Using Barrier Lyapunov Functions for DC Motor System with Time-Varying State Constraints
}

\author{
Lei $\mathrm{Ma}^{1}$ and Dapeng $\mathrm{Li} \mathbb{D}^{2}$ \\ ${ }^{1}$ College of Science, Liaoning University of Technology, Jinzhou, Liaoning 121001, China \\ ${ }^{2}$ School of Electrical Engineering, Liaoning University of Technology, Jinzhou 121001, China \\ Correspondence should be addressed to Dapeng Li; li_dapengsir@163.com
}

Received 28 June 2017; Accepted 16 November 2017; Published 31 January 2018

Academic Editor: Yanan Li

Copyright (C) 2018 Lei Ma and Dapeng Li. This is an open access article distributed under the Creative Commons Attribution License, which permits unrestricted use, distribution, and reproduction in any medium, provided the original work is properly cited.

\begin{abstract}
This paper proposes an adaptive neural network (NN) control approach for a direct-current (DC) system with full state constraints. To guarantee that state constraints always remain in the asymmetric time-varying constraint regions, the asymmetric time-varying Barrier Lyapunov Function (BLF) is employed to structure an adaptive NN controller. As we all know that the constant constraint is only a special case of the time-varying constraint, hence, the proposed control method is more general for dealing with constraint problem as compared with the existing works on DC systems. As far as we know, this system is the first studied situations with timevarying constraints. Using Lyapunov analysis, all signals in the closed-loop system are proved to be bounded and the constraints are not violated. In this paper, the effectiveness of the control method is demonstrated by simulation results.
\end{abstract}

\section{Introduction}

Due to the requirements of practice and the development of theory, the controller design of uncertain system has become a new research direction and attracted more and more scholars' attention. The uncertainty of the actual engineering system has been studied in many works [1-4]. The neural networks [5] and fuzzy logic systems [6] have become the two main tools which can effectively deal with the unknown functions in the systems. In $[7,8]$, these are studies of some actual engineering systems with uncertain parameters. In $[9,10]$, the NN is used to approximate several random perturbations and unknown functions. In [11-16], several nonlinear system solutions are studied based on neural networks and fuzzy logic systems. In [17], adaptive control schemes based on neural networks were proposed for nonlinear systems with unknown functions. Based on neural networks and fuzzy logic systems, the significant studies proposed the novel adaptive tracking control methods for nonlinear SISO systems in [18-20] and MIMO systems in [21-23]. However, it is worth noting that the constraint problem is worth noting in the above approaches, which lead to the inaccuracy or oscillations of the engineering systems and even cause control systems instability.

In fact, there are constraints in most physical systems with various forms, for example, physical stoppages, saturation, performance, and safety specifications, such as restricted robot manipulation system [24], application to chemical process [25], networked surveillance robots systems [26], and nonuniform gantry crane [27]. In recent years, the barrier Lyapunov functions become the main tools to solve the constrained problem which was proposed for the first time in [28]. Based on BLF, some adaptive control methods were presented for nonlinear systems with output constant constraint in $[29,30]$ and state constant constraint in [3134]. As we known, the constant constraint is the special case of the time-varying constraint. Subsequently, the authors in $[35,36]$ proposed some adaptive control approaches to address the stability problem of nonlinear systems with timevarying constraints. 
Motor is the most important electromechanical energy conversion device, which has been widely used in the industrial and agricultural production, transportation, aerospace, and so on. In particular, the motor system with unknown uncertainties has attracted the attention of many scholars, and the control problem of the motor system becomes more and more important. In $[37,38]$, the authors proposed two adaptive control methods for systems with unknown functions. The authors in $[39,40]$ presented an adaptive control with time-varying output constraints for DC motor systems. According to the above descriptions, the urgent problem is how to address the stability problem of the DC motor system with time-varying state constraints.

This paper presents an adaptive NN tracking control method for DC motor systems with time-varying state constraints. As far as we know, there is no work dealing with such DC motor systems in the literature at present stage. The contributions of this paper are summarized as follows. (1) The time-varying state constraints are first considered in the DC motor systems; comparing with the existing on DC motor systems, the proposed control method is more general and extensive in the engineering field. (2) To guarantee that the state constraints always remain in the time-varying constrained sets, the asymmetric time-varying BLF is utilized. (3) A novel adaptive tracking controller based on the neural networks and backstepping technique is structured to guarantee that all signals in the closed-loop system are bounded, the tracking errors converge to a small neighborhood of zero and the time-varying state constraints are not transitioned.

\section{Problem Formulation and Preliminaries}

Consider the dynamic system with the DC motor without vibration mode as the following form:

$$
\begin{aligned}
\dot{\alpha}_{1} & =\alpha_{2} \\
J \dot{\alpha}_{2}+f \dot{\alpha}_{1}+T_{f}+d & =u \\
y & =\alpha_{1},
\end{aligned}
$$

where $\alpha_{1}(t)$ is the motor angular position; $\alpha_{2}$ stands for motor angular velocity; $J$ is a known inertia, $f$ is an unmeasured viscous friction, and $T_{f}$ is an unmeasured nonlinear friction; $d(t)$ represents the unknown disturbance but bounded with $\|d(t)\| \leq d_{M} ; y \in R$ is the system output; and $u$ represents the motor torque. In particular, output $y(t)$ is required as follows:

$$
\underline{k}_{c_{1}}(t)<y(t)<\bar{k}_{c_{1}}(t), \quad \forall t \geq 0
$$

where $\bar{k}_{c_{1}}: R_{+} \rightarrow R$ and $\underline{k}_{c_{1}}: R_{+} \rightarrow R$ such that $\bar{k}_{c_{1}}(t)>\underline{k}_{c_{1}}(t)$,
$\forall t \in R_{+}$. Remark 1. From (2), the states of DC systems are constrained by the considered time-varying functions. In $[35,36]$, the constraint problem is omitted, which is the main factor of the oscillations of the engineering systems. The authors in [39] addressed the stability problem of DC motor systems with constant constraint which is the special case of the time-varying constraint. Comparing with the [40], the authors only consider time-varying output constraint; the proposed adaptive control method tries to stabilize the DC motor systems with time-varying state constraints, which cause the difficulty of controller design.

In this paper, the control objective is to design an adaptive NN tracking controller $u$ which adjusts the output of DC motor systems $y$ to track desired trajectory of the reference signal $y_{d}(t)$ in the range of time-varying constraint functions. Meanwhile, all signals in the closed-loop systems are bounded and the time-varying state constrains are not violated.

Assumption 2 (see [35]). There exist constants $\bar{K}_{c_{i}}$ and $\underline{K}_{c_{i}}, i=$ $0,1, \ldots, n$, such that $\bar{k}_{c_{1}}(t) \leq \bar{K}_{c_{o}}, \underline{k}_{c_{1}}(t) \geq \underline{K}_{c_{0}}$, and $\left|\bar{k}_{c_{1}}^{(i)}(t)\right| \leq$ $\bar{K}_{c_{i}},\left|\underline{k}_{c_{1}}^{(i)}(t)\right| \leq \underline{K}_{c_{i}}, \forall t \geq 0, i=1, \ldots, n$.

Assumption 3 (see [32]). There exist functions $\bar{Y}_{0}: R_{+} \rightarrow R_{+}$ and $\underline{Y}_{0}: R_{+} \rightarrow R_{+}$satisfying $\bar{Y}_{0}(t)<\bar{k}_{c_{1}}(t)$ and $\underline{Y}_{0}(t)>$ $\underline{k}_{c_{1}}(t) \forall t \geq 0$, and positive constants $Y_{i}, i=1, \ldots, n$, such that the desired trajectory $y_{d}(t)$ and its time derivatives satisfy $\underline{Y}_{0}(t) \leq y_{d}(t) \leq \bar{Y}_{0}(t)$ and $\left|y_{d}^{(i)}(t)\right| \leq Y_{i}, i=1, \ldots, n, \forall t \geq 0$.

The following lemma is represented for the establishment of binding compensation and performance limits.

Lemma 4 (see [28]). Let $Z:=\{\xi \in R:|\xi|<1\} \subset R$ and $N:=R^{l} \times Z \subset R^{l+1}$ be open sets. Take into account the system

$$
\dot{\alpha}=g(t, \alpha),
$$

where $\alpha:=[\omega, \xi]^{T} \in \mathcal{N}$ and $g: R_{+} \times \mathcal{N} \rightarrow R^{l+1}$ in $t$ is piecewise continuous and in $\alpha$ is locally Lipschitz, united in $t$, on $R_{+} \times \mathcal{N}$.

Suppose that there are functions $H: R^{l} \times R_{+} \rightarrow R_{+}$ and $V_{1}: Z \rightarrow R_{+}$. In their respective domains, they are continuously differentiable and positive definite, such that

$$
\begin{gathered}
V_{1}(\xi) \rightarrow \infty, \quad|\xi| \longrightarrow 1 \\
\lambda_{1}(\|\omega\|) \leq H(\omega, t) \leq \lambda_{2}(\|\omega\|),
\end{gathered}
$$

where $\lambda_{1}$ and $\lambda_{2}$ are class $K_{\infty}$ functions. Let $V(\alpha):=V_{1}(\xi)+$ $U(\omega, t)$, and $\varepsilon(0) \in Z$. If the inequality is established:

$$
\dot{V}=\frac{\partial V}{\partial \alpha} g \leq 0
$$

in $\xi \in Z, \xi(t) \in Z \forall t \in[0, \infty)$.

Lemma 5 (see [35]). For all $|\xi|<1$ and positive integer $p$, the inequality $\log 1 /\left(1-\xi^{2 p}\right)<\xi^{2 p} /\left(1-\xi^{2 p}\right)$. 
Proof. For $|\xi|<1$, the term $\xi^{2 p} /\left(1-\xi^{2 p}\right)$ can be rewritten as

$$
\begin{aligned}
\frac{\xi^{2 p}}{1-\xi^{2 p}} & =\log \left(e^{\xi^{2 p} /\left(1-\xi^{2 p}\right)}\right) \\
& \geq \log \left[1+\frac{\xi^{2 p}}{1-\xi^{2 p}}+\sum_{n=2}^{\infty} \frac{\left(\xi^{2 p} /\left(1-\xi^{2 p}\right)\right)^{n}}{n !}\right] \\
& \geq \log \left(1+\frac{\xi^{2 p}}{1-\xi^{2 p}}\right)=\log \frac{1}{1-\xi^{2 p}} .
\end{aligned}
$$

The proof is completed.

\section{State Feedback Adaptive Controller Designs}

This paper presents an adaptive tracking controller based on a backstepping technique with the asymmetric time-varying BLF for the DC motor systems. The detailed designs process is shown in this section.

Denote $z_{1}=\alpha_{1}-y_{d}, z_{2}=\alpha_{2}-\sigma_{1}$, where $\sigma_{1}$ is the virtual controller which will be given later on. We consider the timevarying asymmetric BLF:

$$
\begin{aligned}
V_{1}= & \frac{q\left(z_{1}\right)}{2 p} \log \frac{k_{b_{1}}^{2 p}(t)}{k_{b_{1}}^{2 p}(t)-z_{1}^{2 p}} \\
& +\frac{1-q\left(z_{1}\right)}{2 p} \log \frac{k_{a_{1}}^{2 p}(t)}{k_{a_{1}}^{2 p}(t)-z_{1}^{2 p}}
\end{aligned}
$$

where $p$ is a positive integer.

The time-varying barriers are chosen as

$$
\begin{aligned}
& k_{a_{1}}(t)=y_{d}(t)-\underline{k}_{c_{1}}(t) \\
& k_{b_{1}}(t)=\bar{k}_{c_{1}}(t)-y_{d}(t)
\end{aligned}
$$

and $q(\cdot)$ is defined as

$$
q(\cdot)= \begin{cases}1, & \text { if } \cdot>0 \\ 0, & \text { if } \cdot \leq 0\end{cases}
$$

Based on Assumptions 2 and 3, there are positive constants $\underline{k}_{b_{1}}, \bar{k}_{b_{1}}, \underline{k}_{a_{1}}$, and $\bar{k}_{a_{1}}$, such that

$$
\begin{aligned}
& \underline{k}_{b_{1}} \leq k_{b_{1}}(t) \leq \bar{k}_{b_{1}} . \\
& \underline{k}_{a_{1}} \leq k_{a_{1}}(t) \leq \bar{k}_{a_{1}}, \quad \forall t \geq 0
\end{aligned}
$$

Through the change of error coordinates,

$$
\begin{aligned}
\xi_{a_{i}} & =\frac{z_{i}}{k_{a_{i}}}, \\
\xi_{b_{i}} & =\frac{z_{i}}{k_{b_{i}}} \\
\xi_{i} & =q\left(z_{i}\right) \xi_{b_{i}}+\left(1-q\left(z_{i}\right)\right) \xi_{a_{i}}, \quad i=1,2 .
\end{aligned}
$$

According to (12), (7) can be rewritten as

$$
V_{1}=\frac{1}{2 p} \log \frac{1}{1-\xi_{1}^{2 p}} .
$$

Remark 6. According to (10), we know that when $z_{1}>0$, we obtain $q\left(z_{1}\right)=1, \xi_{1}=\xi_{b_{1}}$, and $V_{1}=(1 / 2 p) \log \left(1 /\left(1-\xi_{b_{1}}^{2 p}\right)\right)=$ $(1 / 2 p) \log \left(1 /\left(1-\xi_{1}^{2 p}\right)\right)$. When $z_{1}<0$, we obtain $q\left(z_{1}\right)=0$, $\xi_{1}=\xi_{a_{1}}$, and $V_{1}=(1 / 2 p) \log \left(1 /\left(1-\xi_{a_{1}}^{2 p}\right)\right)=(1 / 2 p) \log (1 /(1-$ $\left.\left.\xi_{1}^{2 p}\right)\right)$. From the above, we can get (13) based on (12).

Obviously, under the premise of $|\xi|<1, V_{1}$ is definite continuously differentiable. The time derivative of $V_{1}$ is

$$
\begin{aligned}
\dot{V}_{1}= & \frac{q\left(z_{1}\right) \xi_{b_{1}}^{2 p-1}}{k_{b_{1}}\left(1-\xi_{b_{1}}^{2 p}\right)}\left(z_{2}+\sigma_{1}-\dot{y}_{d}-z_{1} \frac{\dot{k}_{b_{1}}}{k_{b_{1}}}\right) \\
& +\frac{\left(1-q\left(z_{1}\right)\right) \xi_{a_{1}}^{2 p-1}}{k_{a_{1}}\left(1-\xi_{a_{1}}^{2 p}\right)}\left(z_{2}+\sigma_{1}-\dot{y}_{d}-z_{1} \frac{\dot{k}_{a_{1}}}{k_{a_{1}}}\right) .
\end{aligned}
$$

Choose the virtual controller $\sigma_{1}$ as

$$
\sigma_{1}=-\left(\kappa_{1}+\bar{\kappa}_{1}(t)\right) z_{1}+\dot{y}_{d}-\frac{2 p-1}{2 p} z_{1} .
$$

The time-varying gain is given as

$$
\bar{\kappa}_{i}(t)=\sqrt{\left(\frac{\dot{k}_{a_{i}}}{k_{a_{i}}}\right)^{2}+\left(\frac{\dot{k}_{b_{i}}}{k_{b_{i}}}\right)^{2}+\beta_{i}},
$$

where $\beta_{i}$ and $\kappa_{i}, i=1,2$ are any positive constants. Make sure that the time derivative $\alpha_{1}$ is bounded, when $\dot{k}_{a_{1}}$ and $\dot{k}_{b_{1}}$ are both zero. Substituting (15) and (16) into (14) and noting that

$$
\bar{\kappa}_{i}+q\left(z_{i}\right) \frac{\dot{k}_{b_{i}}}{k_{b_{i}}}+\left(1-q\left(z_{i}\right)\right) \frac{\dot{k}_{a_{i}}}{k_{a_{i}}} \geq 0
$$

we obtain

$$
\begin{aligned}
\dot{V}_{1} & =\left(\frac{q\left(z_{1}\right) \xi_{b_{1}}^{2 p-1}}{k_{b_{1}}\left(1-\xi_{b_{1}}^{2 p}\right)}+\frac{\left(1-q\left(z_{1}\right)\right) \xi_{a_{1}}^{2 p-1}}{k_{a_{1}}\left(1-\xi_{a_{1}}^{2 p}\right)}\right) z_{2} \\
& -z_{1}\left(\bar{\kappa}_{1}(t) \frac{q\left(z_{1}\right) \xi_{b_{1}}^{2 p}}{\left(1-\xi_{b_{1}}^{2 p}\right)}+\bar{\kappa}_{1}(t) \frac{\left(1-q\left(z_{1}\right)\right) \xi_{a_{1}}^{2 p}}{\left(1-\xi_{a_{1}}^{2 p}\right)}\right. \\
& \left.+\frac{\dot{k}_{b_{1}}}{k_{b_{1}}} \frac{q\left(z_{1}\right) \xi_{b_{1}}^{2 p}}{\left(1-\xi_{b_{1}}^{2 p}\right)}+\frac{\dot{k}_{a_{1}}}{k_{a_{1}}} \frac{\left(1-q\left(z_{1}\right)\right) \xi_{a_{1}}^{2 p}}{\left(1-\xi_{a_{1}}^{2 p}\right)}\right) \\
& -\kappa_{1} z_{1}\left(\frac{q\left(z_{1}\right) \xi_{b_{1}}^{2 p}}{\left(1-\xi_{b_{1}}^{2 p}\right)}+\frac{\left(1-q\left(z_{1}\right)\right) \xi_{a_{1}}^{2 p}}{\left(1-\xi_{a_{1}}^{2 p}\right)}\right)-\frac{2 p-1}{2 p} \\
& \cdot \mu_{1} z_{1}^{2 p}
\end{aligned}
$$


where

$$
\mu_{1}=\frac{q\left(z_{1}\right)}{k_{b_{1}}^{2 p}-z_{1}^{2 p}}+\frac{1-q\left(z_{1}\right)}{k_{a_{1}}^{2 p}-z_{1}^{2 p}} .
$$

After finishing it, we get

$$
\begin{aligned}
\dot{V}_{1} & =\left(\frac{q\left(z_{1}\right)}{\left(k_{b_{1}}^{2 p}-z_{1}^{2 p}\right)}+\frac{\left(1-q\left(z_{1}\right)\right)}{\left(k_{a_{1}}^{2 p}-z_{1}^{2 p}\right)}\right) z_{1}^{2 p-1} z_{2} \\
& -\frac{\xi_{1}^{2 p}}{\left(1-\xi_{1}^{2 p}\right)}\left(\frac{\dot{k}_{b_{1}}}{k_{b_{1}}} q\left(z_{1}\right)+\frac{\dot{k}_{a_{1}}}{k_{a_{1}}}\left(1-q\left(z_{1}\right)\right)\right. \\
& \left.+\bar{\kappa}_{1}(t)\right)-\frac{\kappa_{1} \xi_{1}^{2 p}}{\left(1-\xi_{1}^{2 p}\right)}-\frac{2 p-1}{2 p} \mu_{1} z_{1}^{2 p} .
\end{aligned}
$$

Based on (12), we obtain

$$
\dot{V}_{1} \leq-\frac{\kappa_{1} \xi_{1}{ }^{2 p}}{1-\xi_{1}{ }^{2 p}}+\mu_{1} z_{1}{ }^{2 p-1} z_{2}-\frac{2 p-1}{2 p} \mu_{1} z_{1}^{2 p} .
$$

Using Young's inequality, the following inequality holds:

$$
\mu_{1} z_{1}^{2 p-1} z_{2} \leq \mu_{1}\left(\frac{2 p-1}{2 p} z_{1}^{2 p}+\frac{1}{2 p} z_{2}^{2 p}\right) .
$$

Substituting (22) into (21), $\dot{V}_{1}$ can be further rewritten as

$$
\dot{V}_{1} \leq-\frac{\kappa_{1} \xi_{1}^{2 p}}{1-\xi_{1}^{2 p}}+\frac{1}{2 p} \mu_{1} z_{2}^{2 p}
$$

The Barrier Lyapunov Function $V_{2}$ is given as

$$
\begin{aligned}
V_{2}= & V_{1}+\frac{q\left(z_{2}\right)}{2 p} \log \frac{k_{b_{2}}^{2 p}(t)}{k_{b_{2}}^{2 p}(t)-z_{2}^{2 p}} \\
& +\frac{1-q\left(z_{2}\right)}{2 p} \log \frac{k_{a_{2}}^{2 p}(t)}{k_{a_{2}}^{2 p}(t)-z_{2}^{2 p}} .
\end{aligned}
$$

Then, differentiating of $V_{2}$ with respect to time is given by

$$
\begin{aligned}
\dot{V}_{2}= & \dot{V}_{1}+\frac{q\left(z_{2}\right) \xi_{b_{2}}^{2 p-1}}{k_{b_{2}}\left(1-\xi_{b_{2}}^{2 p}\right)}\left(\dot{z}_{2}-z_{2} \frac{\dot{k}_{b_{2}}}{k_{b_{2}}}\right) \\
& +\frac{\left(1-q\left(z_{2}\right)\right) \xi_{a_{2}}^{2 p-1}}{k_{a_{2}}\left(1-\xi_{a_{2}}^{2 p}\right)}\left(\dot{z}_{2}-z_{2} \frac{\dot{k}_{a_{2}}}{k_{a_{2}}}\right) .
\end{aligned}
$$

From the definition of the tracking error $z_{2}=\alpha_{2}-\sigma_{1}$, it is easy to obtain $\dot{z}_{2}=\dot{\alpha}_{2}-\dot{\sigma}_{1}$, and the derivative of the virtual controller is given as

$$
\dot{\sigma}_{1}=\frac{\partial \sigma_{1}}{\partial \alpha_{1}} \alpha_{2}+\sum_{j=0}^{1} \frac{\partial \sigma_{1}}{\partial \zeta_{1}} \zeta_{1}^{(j+1)}
$$

where $\zeta_{1}=\left[y_{d}, k_{a_{1}}, k_{b_{1}}\right]^{T}$.
According to (26), (25) can be rewritten as

$\dot{V}_{2}$

$$
\begin{aligned}
= & \left(\dot{\alpha}_{2}-\dot{\sigma}_{1}\right)\left(\frac{1-q\left(z_{2}\right)}{k_{a_{2}}} \frac{\xi_{a_{2}}^{2 p-1}}{1-\xi_{a_{2}}^{2 p}}+\frac{q\left(z_{2}\right)}{k_{b_{2}}} \frac{\xi_{b_{2}}^{2 p-1}}{1-\xi_{b_{2}}^{2 p}}\right) \\
& -z_{2} \frac{\dot{k}_{b_{2}}}{k_{b_{2}}} \frac{q\left(z_{2}\right)}{k_{b_{2}}} \frac{\xi_{b_{2}}^{2 p-1}}{1-\xi_{b_{2}}^{2 p}} \\
& -z_{2} \frac{\dot{k}_{a_{2}}}{k_{a_{2}}} \frac{1-q\left(z_{2}\right)}{k_{a_{2}}} \frac{\xi_{a_{2}}^{2 p-1}}{1-\xi_{a_{2}}^{2 p}}+\dot{V}_{1} .
\end{aligned}
$$

Based on (23), we get

$$
\begin{aligned}
\dot{V}_{2} \leq & -\frac{\kappa_{1} \xi_{1}^{2 p}}{1-\xi_{1}^{2 p}}+\frac{1}{2 p} \mu_{1} z_{2}^{2 p}+\left(\dot{\alpha}_{2}-\dot{\sigma}_{1}\right) \frac{\xi_{2}^{2 p}}{z_{2}\left(1-\xi_{2}^{2 p}\right)} \\
& -z_{2} \frac{\dot{k}_{b_{2}}}{k_{b_{2}}} \frac{q\left(z_{2}\right)}{k_{b_{2}}} \frac{\xi_{b_{2}}^{2 p-1}}{1-\xi_{b_{2}}^{2 p}} \\
& -z_{2} \frac{\dot{k}_{a_{2}}}{k_{a_{2}}} \frac{1-q\left(z_{2}\right)}{k_{a_{2}}} \frac{\xi_{a_{2}}^{2 p-1}}{1-\xi_{a_{2}}^{2 p}} .
\end{aligned}
$$

Substituting (26) into the above formula, we obtain

$$
\begin{aligned}
\dot{V}_{2}= & -\frac{\kappa_{1} \xi_{1}^{2 p}}{1-\xi_{1}^{2 p}} \\
& +\frac{\xi_{2}^{2 p}}{z_{2}\left(1-\xi_{2}^{2 p}\right)}\left(J^{-1}\left(-f \dot{\alpha}_{1}-T_{f}+u\right)-\dot{\sigma}_{1}\right) \\
& -\frac{q\left(z_{2}\right)}{k_{b_{2}}} \frac{\xi_{b_{2}}^{2 p-1}}{1-\xi_{b_{2}}^{2 p}}\left(z_{2} \frac{\dot{k}_{b_{2}}}{k_{b_{2}}}\right) \\
& -\frac{1-q\left(z_{2}\right)}{k_{a_{2}}} \frac{\xi_{a_{2}}^{2 p-1}}{1-\xi_{a_{2}}^{2 p}}\left(z_{2} \frac{\dot{k}_{a_{2}}}{k_{a_{2}}}\right)-\frac{J^{-1} \xi_{2}^{2 p}}{z_{2}\left(1-\xi_{2}^{2 p}\right)} d \\
& +\frac{1}{2 p} \mu_{1} z_{2}^{2 p} .
\end{aligned}
$$

Using Young's inequality and noting $\|d(t)\| \leq d_{M}$, we obtain

$$
-\frac{J^{-1} \xi_{2}^{2 p}}{z_{2}\left(1-\xi_{2}^{2 p}\right)} d \leq \frac{1}{2 \gamma_{1}}\left(\frac{J^{-1} \xi_{2}^{2 p}}{z_{2}\left(1-\xi_{2}^{2 p}\right)}\right)^{2}+\frac{1}{2} \gamma_{1} d_{M}^{2},
$$

where $\gamma_{1}$ is a positive constant. 
Based on (30), (29) can be rewritten as

$$
\begin{aligned}
\dot{V}_{2}= & -\frac{\kappa_{1} \xi_{1}^{2 p}}{1-\xi_{1}^{2 p}}+\frac{1}{2 p} \mu_{1} z_{2}^{2 p}+\frac{\xi_{2}^{2 p}}{z_{2}\left(1-\xi_{2}^{2 p}\right)} \\
& \times\left(J^{-1}\left(-f \dot{\alpha}_{1}-T_{f}+u\right)-\dot{\sigma}_{1}\right)+\frac{q\left(z_{2}\right)}{k_{b_{2}}} \frac{\xi_{b_{2}}^{2 p-1}}{1-\xi_{b_{2}}^{2 p}} \\
& \times\left(-z_{2} \frac{\dot{k}_{b_{2}}}{k_{b_{2}}}\right)+\frac{1-q\left(z_{2}\right)}{k_{a_{2}}} \frac{\xi_{a_{2}}^{2 p-1}}{1-\xi_{a_{2}}^{2 p}}\left(-z_{2} \frac{\dot{k}_{a_{2}}}{k_{a_{2}}}\right) \\
& +\frac{1}{2 \gamma_{1}}\left(\frac{J^{-1} \xi_{2}^{2 p}}{z_{2}\left(1-\xi_{2}^{2 p}\right)}\right)^{2}+\frac{1}{2} \gamma_{1} d_{M}^{2} .
\end{aligned}
$$

For convenience, we define

$$
M(Z)=J^{-1}\left(T_{f}+f \dot{\alpha}_{1}\right)+\dot{\sigma}_{1} .
$$

In fact, since the parameters of $f$ and $T_{f}$ are not available, $M$ is unknown in practice. In order to solve the uncertainty of this parameter, we designed $\mathrm{NN}$, as shown below to estimate

$$
M(Z)=W^{* T} S(Z)+\varepsilon(Z),
$$

where $Z=\left[\alpha_{1}^{T}, \alpha_{2}^{T}\right]^{T} \in \Omega_{z} \subset R^{3}$, and similar to [28], we assume that the approximate error $\varepsilon(Z)$ satisfies $|\varepsilon(Z)| \leq \varepsilon^{*}$ with the constant $\varepsilon^{*}>0$.

Substituting (33), (31) can be rewritten as

$$
\begin{aligned}
\dot{V}_{2} \leq & -\frac{\kappa_{1} \xi_{1}^{2 p}}{1-\xi_{1}^{2 p}}+\frac{1}{2 p} \mu_{1} z_{2}^{2 p}+\frac{J^{-1} \xi_{2}^{2 p} \widetilde{W}^{T} S(Z)}{z_{2}\left(1-\xi_{2}^{2 p}\right)} \\
& -\frac{J^{-1} \xi_{2}^{2 p}}{z_{2}\left(1-\xi_{2}^{2 p}\right)} \varepsilon(Z)+\frac{1}{2 \gamma_{1}}\left(\frac{J^{-1} \xi_{2}^{2 p}}{z_{2}\left(1-\xi_{2}^{2 p}\right)}\right)^{2} \\
& +\frac{1}{2} \gamma_{1} d_{M}^{2}+\bar{\kappa}_{2} \frac{\xi_{2}^{2 p}}{1-\xi_{2}^{2 p}}+\frac{J^{-1} \xi_{2}^{2 p}}{z_{2}\left(1-\xi_{2}^{2 p}\right)} u
\end{aligned}
$$

According to Young's inequality, we can easily obtain

$$
-\frac{J^{-1} \xi_{2}^{2 p} \varepsilon(Z)}{z_{2}\left(1-\xi_{2}^{2 p}\right)} \leq \frac{1}{2 \gamma_{2}}\left(\frac{J^{-1} \xi_{2}^{2 p}}{z_{2}\left(1-\xi_{2}^{2 p}\right)}\right)^{2}+\frac{1}{2} \gamma_{2} \varepsilon^{* 2},
$$

where $\gamma_{2}$ is a positive constant.

Based on (35), (34) can be rewritten as

$$
\begin{aligned}
\dot{V}_{2} \leq & -\frac{\kappa_{1} \xi_{1}^{2 p}}{1-\xi_{1}^{2 p}}+\frac{1}{2 p} \mu_{1} z_{2}^{2 p}+\frac{J^{-1} \xi_{2}^{2 p} \widetilde{W}^{T} S(Z)}{z_{2}\left(1-\xi_{2}^{2 p}\right)} \\
& +\frac{1}{2 \gamma_{2}}\left(\frac{J^{-1} \xi_{2}^{2 p}}{z_{2}\left(1-\xi_{2}^{2 p}\right)}\right)^{2}+\frac{1}{2} \gamma_{2} \varepsilon^{* 2}+\bar{\kappa}_{2} \frac{\xi_{2}^{2 p}}{1-\xi_{2}^{2 p}}
\end{aligned}
$$

$$
\begin{aligned}
& +\frac{1}{2 \gamma_{1}}\left(\frac{J^{-1} \xi_{2}^{2 p}}{z_{2}\left(1-\xi_{2}^{2 p}\right)}\right)^{2}+\frac{1}{2} \gamma_{1} d_{M}^{2} \\
& +\frac{J^{-1} \xi_{2}^{2 p}}{z_{2}\left(1-\xi_{2}^{2 p}\right)} u .
\end{aligned}
$$

The actual controller is given as

$$
\begin{aligned}
u= & -\frac{1}{2 p} J \mu_{1} z_{2}\left(k_{b_{2}}^{2 p}-z_{2}^{2 p}\right)-\frac{J^{-1} \xi_{2}^{2 p}}{2 \gamma_{1} z_{2}\left(1-\xi_{2}^{2 p}\right)} \\
& -\frac{J^{-1} \xi_{2}^{2 p}}{2 \gamma_{2} z_{2}\left(1-\xi_{2}^{2 p}\right)}+J z_{2} \widehat{W}^{T} S(Z) \\
& -J z_{2}\left(\kappa_{2}+\bar{\kappa}_{2}\right) .
\end{aligned}
$$

Substituting (37), we obtain

$$
\begin{aligned}
\dot{V}_{2} \leq & -\frac{\kappa_{1} \xi_{1}^{2 p}}{1-\xi_{1}^{2 p}}-\kappa_{2} \frac{\xi_{2}^{2 p}}{\left(1-\xi_{2}^{2 p}\right)}+\frac{1}{2} \gamma_{1} d_{M}^{2} \\
& +\frac{\xi_{2}^{2 p}}{1-\xi_{2}^{2 p}} \widetilde{W}^{T} S(Z)+\frac{1}{2} \gamma_{2} \varepsilon^{* 2} .
\end{aligned}
$$

Design the Lyapunov function candidate $V_{3}$ :

$$
V_{3}=V_{2}+\frac{1}{2} \widetilde{W}^{T} \Gamma^{-1} \widetilde{W}
$$

where $\Gamma=\Gamma^{-1}>0$ is a constant matrix and $\widetilde{W}=\widehat{W}-W^{*}$.

The time derivative of $V_{3}$ is

$$
\dot{V}_{3}=\dot{V}_{2}+\widetilde{W}^{T} \Gamma^{-1} \dot{\widehat{W}} .
$$

Based on (38), we obtain

$$
\begin{aligned}
\dot{V}_{3} \leq & -\frac{\kappa_{1} \xi_{1}^{2 p}}{1-\xi_{1}^{2 p}}-\kappa_{2} \frac{\xi_{2}^{2 p}}{\left(1-\xi_{2}^{2 p}\right)}+\frac{1}{2} \gamma_{1} d_{M}^{2}+\frac{1}{2} \gamma_{2} \varepsilon^{* 2} \\
& +\widetilde{W}^{T} \Gamma^{-1} \dot{W}+\frac{\xi_{2}^{2 p}}{1-\xi_{2}^{2 p}} \widetilde{W}^{T} S(Z) .
\end{aligned}
$$

The adaptive law is given as follows:

$$
\dot{\widehat{W}}=\Gamma\left(-\frac{\xi_{2}^{2 p}}{1-\xi_{2}^{2 p}} S(Z)-\eta \widehat{W}\right),
$$

where $\eta$ is a positive constant.

Substituting (42) into (41), we get

$$
\begin{aligned}
\dot{V}_{3}= & -\frac{\kappa_{1} \xi_{1}^{2 p}}{1-\xi_{1}^{2 p}}-\kappa_{2} \frac{\xi_{2}^{2 p}}{\left(1-\xi_{2}^{2 p}\right)}+\frac{1}{2} \gamma_{1} d_{M}^{2}+\frac{1}{2} \gamma_{2} \varepsilon^{* 2} \\
& -\widetilde{W}^{T} \eta\left(\widetilde{W}+W^{*}\right) .
\end{aligned}
$$


Using Young's inequality,

$$
\begin{aligned}
\dot{V}_{3} \leq & -\frac{\kappa_{1} \xi_{1}^{2 p}}{1-\xi_{1}^{2 p}}-\kappa_{2} \frac{\xi_{2}^{2 p}}{\left(1-\xi_{2}^{2 p}\right)}+\frac{1}{2} \gamma_{1} d_{M}^{2}+\frac{1}{2} \gamma_{2} \varepsilon^{* 2} \\
& -\frac{\eta}{2}\left(\|\widetilde{W}\|^{2}-\left\|W^{*}\right\|^{2}\right) .
\end{aligned}
$$

After finishing it, we get

$$
\begin{aligned}
\dot{V}_{3} \leq & -\kappa_{1} \log \frac{1}{1-\xi_{1}^{2 p}}-\frac{\eta}{2}\|\widetilde{W}\|^{2}+\frac{\eta}{2}\left\|W^{*}\right\|^{2} \\
& -\kappa_{2} \log \frac{1}{1-\xi_{2}{ }^{2 p}}+\frac{1}{2} \gamma_{1} d_{M}^{2}+\frac{1}{2} \gamma_{2} \varepsilon^{* 2} .
\end{aligned}
$$

Then, the above inequality can be rewritten as

$$
\dot{V}_{3} \leq-\rho V_{3}+C
$$

where

$$
\begin{aligned}
& \rho=\min \left\{2 \kappa_{1}, 2 \kappa_{2}, \eta \Gamma^{-1}\right\} . \\
& C=\frac{1}{2} \gamma_{1} d_{M}^{2}+\frac{1}{2} \gamma_{2} \varepsilon^{* 2}+\frac{\eta}{2}\left\|W^{*}\right\|^{2}
\end{aligned}
$$

Theorem 7. Consider the unknown DC motor control system (1), based on the assumptions of Assumptions 2 and 3, Lemma 4, actual controller (37), and the adaptive law (42). The following properties guaranteed that the tracking error singles will remain in a compact neighborhood of zero, that is, $\lim _{t \rightarrow \infty}\left|y(t)-y_{d}(t)\right|=0$, all signals of the closed-loop system are bounded, and all state constraints are never violated.

Proof. With both sides of (46) multiplied by $e^{\rho t}$, we obtain

$$
\frac{d}{d t}\left(V_{3} e^{\rho t}\right) \leq C e^{\rho t}
$$

Integrating $(48)$ over $[0, t]$, we have

$$
0 \leq V_{3}(t) \leq V_{3}(0) e^{-\rho t}+\frac{C}{\rho} .
$$

Based on (7), (24), and (39), we can obtain

$$
\begin{aligned}
V_{3}= & \frac{1}{2} \log \frac{k_{b_{1}}^{2 p}(t)}{k_{b_{1}}^{2 p}(t)-z_{1}^{2 p}}+\frac{1}{2} \log \frac{k_{b_{2}}^{2 p}(t)}{k_{b_{2}}^{2 p}(t)-z_{2}^{2 p}} \\
& +\frac{1}{2} \widetilde{W}^{T} \Gamma^{-1} \widetilde{W} .
\end{aligned}
$$

Then, we have

$$
\frac{1}{2} \log \frac{k_{b_{1}}^{2 p}(t)}{k_{b_{1}}^{2 p}(t)-z_{1}^{2 p}} \leq V_{3 A}(t) \leq V_{3 A}(0),
$$

where

$$
\begin{aligned}
V_{3 A}(0)= & \frac{1}{2} \log \frac{k_{b_{1}}^{2 p}(0)}{k_{b_{1}}^{2 p}(0)-z_{1}^{2 p}} \\
& +\frac{1}{4} \lambda_{\max }\left(\Gamma^{-1}\right)\left\|\widehat{W}(0)-W^{*}\right\|+\frac{C}{2 \rho} .
\end{aligned}
$$

Therefore, we know that

$$
\left(\frac{z_{1}}{k_{b}(t)}\right)^{2 p} \leq 1-e^{-2 V_{3 A}(0)} .
$$

Based on the above inequality, the following inequality is obtained:

$$
\left|z_{1}(t)\right| \leq D_{1}(t)
$$

where

$$
D_{1}(t)=k_{b_{1}}(t) \sqrt[2 p]{1-e^{-2 V_{3 A}(0)}}
$$

Similar to the derivation of $z_{1}$, we can obtain the conclusion that

$$
\left|z_{2}(t)\right| \leq D_{2}(t)
$$

where

$$
D_{2}(t)=k_{b_{2}}(t) \sqrt[2 p]{1-e^{-2 V_{3 B}(0)}}
$$

From Assumption 2, we can be known that $\left|\alpha_{1}(0)\right|<$ $K_{c_{1}}(0)$, and from the definition of $k_{c_{1}}(t)$, we have $\left|z_{1}(t)\right|<$ $k_{b_{1}}(t)$. In fact, from $\alpha_{1}=z_{1}+y_{d}$ and $\alpha_{2}=z_{2}+\sigma_{1}$, we obtain

$$
\left|\alpha_{1}(t)\right|<k_{b_{1}}(t)+y_{d}(t)
$$

Based on the above inequality, we know $|y(t)| \leq k_{c_{1}}(t)$, $\forall t \geq 0$. Therefore, the output signals are bounded.

Obviously, we can clearly obtain that the virtual controller $\sigma_{1}$ is bounded in (15). Based on $z_{2}=\alpha_{2}-\sigma_{1}$ and (56), $\alpha_{2}$ is bounded. In addition, from (37) and (42), we know the actual controller $u$ and the adaptive law $\widehat{W}$ are bounded. Therefore, all the closed-loop system signals are bounded.

The proof is completed.

Remark 8. In the above analysis, it is apparent that the boundedness of $z_{1}$ lies on the design parameters $\gamma_{1}, \gamma_{2}, \eta$, $d_{M}, \varepsilon^{*}, W^{*}, \kappa_{1}, \kappa_{2}$, and $\Gamma^{-1}$. If we fix $\eta>0$, it is clear that decreasing $\gamma_{i}$ might result in small $C$ and increasing $\kappa_{i}$ might result in large $\rho$; thus, it will help to reduce $C / \rho$. This represents that the tracking errors can be made arbitrarily small by selecting the design parameters appropriately.

\section{Simulation Results}

To illustrate the validity of the proposed adaptive NN control method, a simulation example is provided. Specifically, the following the DC motor system is described by

$$
J \dot{\alpha}_{2}+f \dot{\alpha}_{1}+T_{f}+d=u,
$$

where the inertia is $J=0.018 \mathrm{~kg} \cdot \mathrm{m}^{2}, f$ denotes an unmeasured viscous friction with $f=\sin (t)+1.82, T_{f}$ is an unmeasured nonlinear friction with $T_{f}=0.987$, and $d$ is the external interference with $d=0.05 \sin t$. The desired reference signal is given as $y_{d}=0.4 \cos t-0.2$. The virtual 


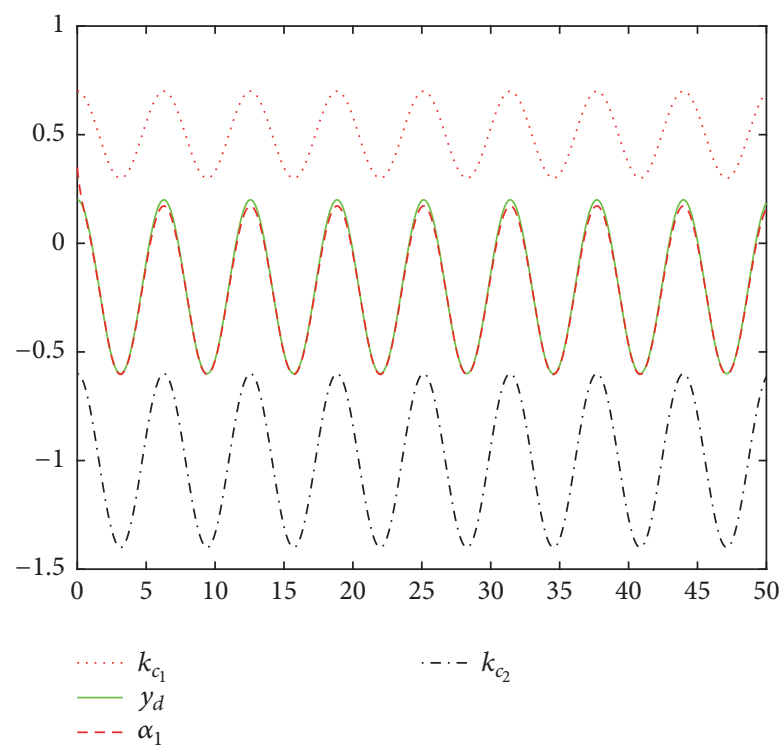

FIGURE 1: The trajectories of output $\alpha_{1}$ and the reference signal $y_{d}(t)$.

controller, the actual controller, and the adaptation law are chosen as follows:

$$
\begin{aligned}
\sigma_{1}= & -\left(\kappa_{1}+\bar{\kappa}_{1}(t)\right) z_{1}+\dot{y}_{d}-\frac{2 p-1}{2 p} z_{1} \\
u= & -\frac{1}{2 p} J \mu_{1} z_{2}\left(k_{b_{2}}^{2 p}-z_{2}^{2 p}\right)-J\left(\kappa_{2}+\bar{\kappa}_{2}\right) z_{2} \\
& -\frac{J^{-1} \xi_{2}^{2 p}}{2 \gamma_{1} z_{2}\left(1-\xi_{2}^{2 p}\right)}-\frac{J^{-1} \xi_{2}^{2 p}}{2 \gamma_{2} z_{2}\left(1-\xi_{2}^{2 p}\right)} \\
& +J z_{2} \widehat{W}^{T} S(Z) \\
\dot{\widehat{W}}= & \Gamma\left(-\frac{\xi_{2}^{2 p}}{1-\xi_{2}^{2 p}} S(Z)-\eta \widehat{W}\right) .
\end{aligned}
$$

The angular position and the angular velocity of motor systems are bounded by $k_{c_{1}}<\alpha_{1}<k_{c_{2}}$ and $k_{c_{3}}<\alpha_{2}<k_{c_{4}}$ with $k_{c_{1}}=0.5+0.2 \cos (t), k_{c_{2}}=-1+0.4 \cos (t), k_{c_{3}} \stackrel{c_{4}}{=}$ $-1.4+0.4 \cos (t)$, and $k_{c_{4}}=0.6+0.2 \cos (t)$. The NN $W^{T} S(z)$ contains 20 nodes and the centers $\mu_{i}, i=1, \ldots, 20$. The design parameters of the proposed control method are chosen as $\Gamma=2.5 I, p=2, \beta_{1}=4, \beta_{2}=4, \kappa_{1}=2, \kappa_{2}=2, c=1$, and $T_{f}=0.987$ and the initial condition of the system state is chosen as $\alpha_{1}(0)=0.35, \alpha_{2}(0)=-0.95$, and $\widehat{W}(0)=0$.

For the DC motor system, using a method of controlling the program can be obtained by the simulation results shown in Figures 1-5. Figures 1 and 2 show the output trajectory. Figure 1 shows the output and the reference signal tracking effect; the figure shows that the two curves almost coincide; that is to say, the tracking error converges to zero. Figure 3 shows the tracking error trajectory of $z_{1}(t)$ initially from the boundaries $k_{b_{1}}(t)$ and $-k_{a_{1}}(t)$ repulsion, but eventually converging to zero. Figure 4 shows a bounded and adaptive law of locus. According to Figure 4, we can see that the track

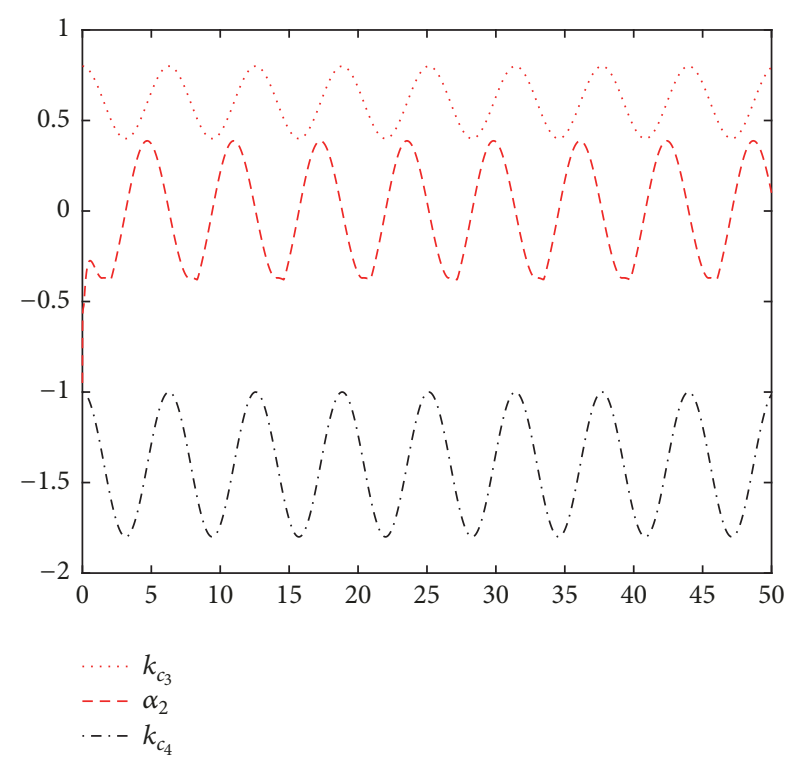

FIGURE 2: Trajectory of state $\alpha_{2}$.

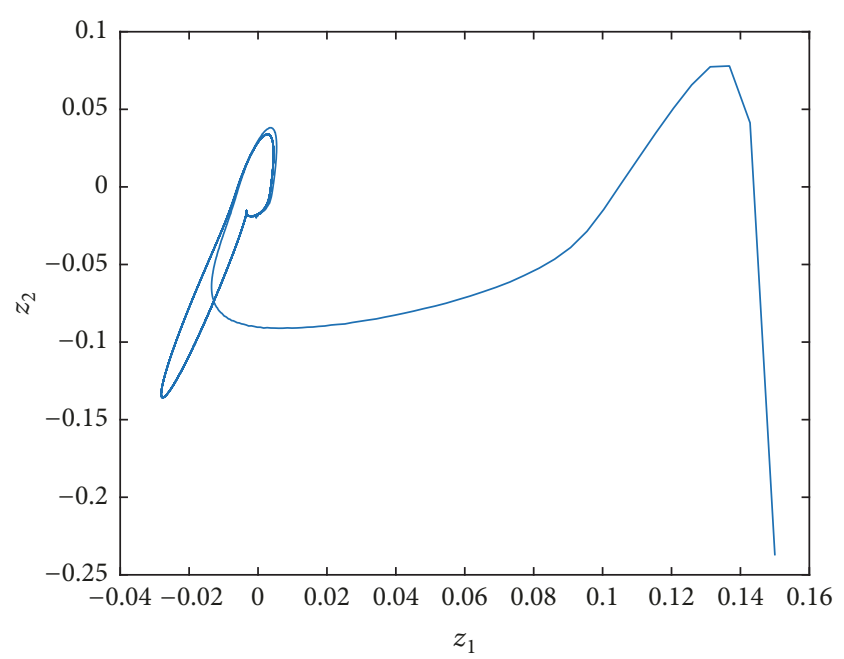

FIGURE 3: Phase portrait of $z_{1}$ and $z_{2}$.

adaptation law is bounded. Thus, we can conclude that a good tracking performance can make all the signals in the closedloop system bounded. From Figure 5, it can be observed that the control input is bounded by a bounded back and forth reciprocate.

\section{Conclusion}

In this paper, we propose an adaptive tracking control method for a DC system with full state constraints. The asymmetric time-varying BLF is employed to guarantee that the states always remain in the time-varying constrained sets. In the asymmetric system, neural networks and a backstepping technique are used to construct an adaptive control and adaptation laws to ensure that all signals in the closed-loop system are bounded and the state constraints are 


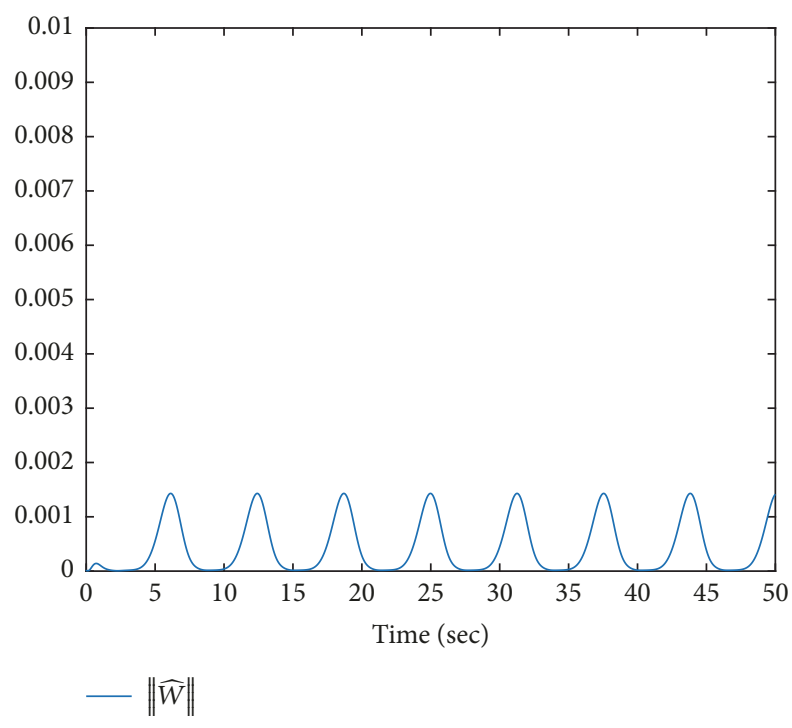

FIGURE 4: The trajectory of $\|\widehat{W}\|$.

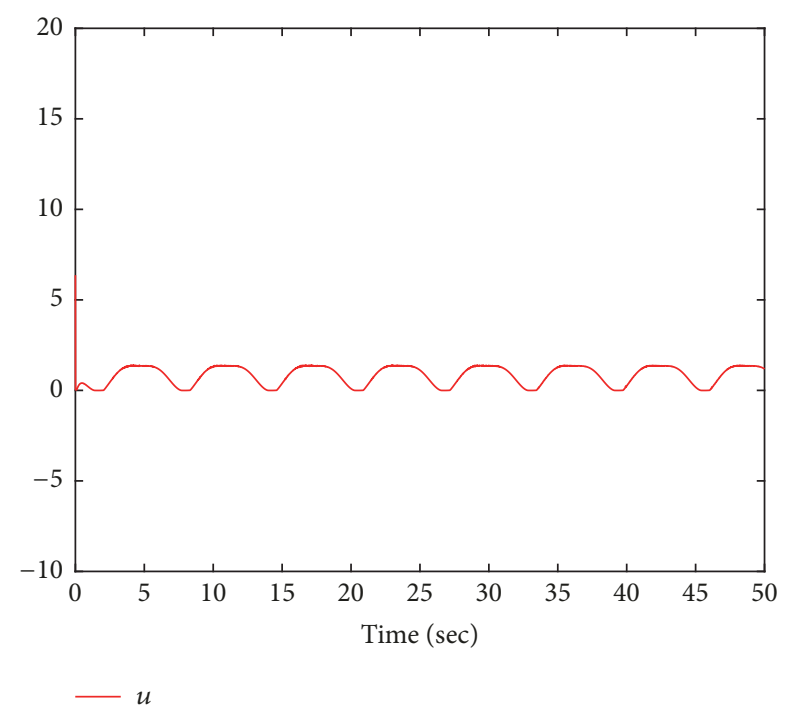

Figure 5: The trajectory of input $u$.

not transitioned. The performances of the adaptive control method based asymmetric time-varying BLF are verified by a simulation example.

\section{Conflicts of Interest}

The authors declare that there are no conflicts of interest regarding the publication of this paper.

\section{Acknowledgments}

This work was supported by the National Natural Science Foundation of China (61603164, 61473139, and 61622303) and the project for Distinguished Professor of Liaoning Province.

\section{References}

[1] F. Deng, S. Guo, R. Zhou, and J. Chen, "Sensor Multifault Diagnosis with Improved Support Vector Machines," IEEE Transactions on Automation Science and Engineering, vol. 14, no. 2, pp. 1053-1063, 2017.

[2] F. Deng, S. Guan, X. Yue et al., "Energy-Based Sound Source Localization with Low Power Consumption in Wireless Sensor Networks," IEEE Transactions on Industrial Electronics, vol. 64, no. 6, pp. 4894-4902, 2017.

[3] L. Chen and Q. Wang, "Adaptive robust control for a class of uncertain MIMO non-affine nonlinear systems," IEEE/CAA Journal of Automatica Sinica, vol. 3, no. 1, pp. 105-112, 2016.

[4] M. Chen, "Robust tracking control for self-balancing mobile robots using disturbance observer," IEEE/CAA Journal of Automatica Sinica, vol. 4, no. 3, pp. 458-465, 2017.

[5] P. Liu, Z. Zeng, and J. Wang, "Multiple Mittag-Leffler Stability of Fractional-Order Recurrent Neural Networks," IEEE Transactions on Systems, Man, and Cybernetics: Systems, vol. 47, no. 8, pp. 2279-2288, 2017.

[6] Q. Shen, B. Jiang, and V. Cocquempot, "Fuzzy logic systembased adaptive fault-tolerant control for near-space vehicle attitude dynamics with actuator faults," IEEE Transactions on Fuzzy Systems, vol. 21, no. 2, pp. 289-300, 2013.

[7] L. Liu, Z. Wang, and H. Zhang, "Adaptive fault-tolerant tracking control for MIMO discrete-time systems via reinforcement learning algorithm with less learning parameters," IEEE Transactions on Automation Science and Engineering, vol. 14, no. 1, pp. 299-313, 2017.

[8] Z. Li, H. Xiao, C. Yang, and Y. Zhao, "Model predictive control of nonholonomic chained systems using general projection neural networks optimization," IEEE Transactions on Systems, Man, and Cybernetics: Systems, vol. 45, no. 10, pp. 1313-1321, 2015.

[9] J. Li, W. S. Chen, and J.-M. Li, "Adaptive neural network output-feedback decentralized stabilization for a class of largescale stochastic nonlinear strict-feedback systems," International Journal of Robust and Nonlinear Control, vol. 21, no. 3, pp. 452-472, 2011.

[10] W. S. Chen, L. C. Jiao, and Z. B. Du, "Output-feedback adaptive dynamic surface control of stochastic non-linear systems using neural network," IET Control Theory \& Applications, vol. 4, no. 12, pp. 3012-3021, 2010.

[11] Q. Zhou, H. Li, L. Wang, and R. Lu, "Prescribed Performance Observer-Based Adaptive Fuzzy Control for NonstrictFeedback Stochastic Nonlinear Systems," IEEE Transactions on Systems, Man, and Cybernetics: Systems, 2017.

[12] B. Xu, Z. Shi, C. Yang, and F. Sun, "Composite neural dynamic surface control of a class of uncertain nonlinear systems in strict-feedback form," IEEE Transactions on Cybernetics, vol. 44, no. 12, pp. 2626-2634, 2014.

[13] W. Chen and L. Jiao, "Adaptive tracking for periodically timevarying and nonlinearly parameterized systems using multilayer neural networks," IEEE Transactions on Neural Networks and Learning Systems, vol. 21, no. 2, pp. 348-351, 2010.

[14] T. Wang, H. Gao, and J. Qiu, "A combined adaptive neural network and nonlinear model predictive control for multirate networked industrial process control," IEEE Transactions on Neural Networks and Learning Systems, vol. 27, no. 2, pp. 416425, 2016.

[15] B. Chen, C. Lin, X. P. Liu, and K. F. Liu, "Observer-based adaptive fuzzy control for a class of nonlinear delayed systems," 
IEEE Transactions on Systems, Man, and Cybernetics: Systems, vol. 46, no. 1, pp. 27-36, 2016.

[16] L. Wang, M. Basin, H. Li, and R. Lu, "Observer-based Composite Adaptive Fuzzy Control for Nonstrict-feedback Systems with Actuator Failures," IEEE Transactions on Fuzzy Systems, no. 99, pp. 1-1, 2017.

[17] Z. Liu, C. Chen, Y. Zhang, and C. L. P. Chen, "Adaptive neural control for dual-arm coordination of humanoid robot with unknown nonlinearities in output mechanism," IEEE Transactions on Cybernetics, vol. 45, no. 3, pp. 521-532, 2015.

[18] A. Boulkroune, "A fuzzy adaptive control approach for nonlinear systems with unknown control gain sign," Neurocomputing, vol. 179, pp. 318-325, 2016.

[19] S. C. Tong, L. L. Zhang, and Y. M. Li, "Observed-based adaptive fuzzy decentralized tracking control for switched uncertain nonlinear large-scale systems with dead zones," IEEE Transactions on Systems, Man, and Cybernetics: Systems, vol. 46, no. 1, pp. 37-47, 2016.

[20] N. Shirkhani, M. A. Khanesar, and M. Teshnehlab, "Indirect model reference fuzzy control of SISO fractional order nonlinear chaotic systems," Procedia Computer Science, vol. 102, pp. 309-316, 2016.

[21] C. Yang, Y. Jiang, Z. Li, W. He, and C.-Y. Su, "Neural control of bimanual robots with guaranteed global stability and motion precision," IEEE Transactions on Industrial Informatics, vol. 13, no. 3, pp. 1162-1171, 2017.

[22] S. S. Ge and C. Wang, "Adaptive neural control of uncertain MIMO nonlinear systems," IEEE Transactions on Neural Networks and Learning Systems, vol. 15, no. 3, pp. 674-692, 2004.

[23] S. C. Tong, S. Sui, and Y. Li, "Fuzzy adaptive output feedback control of MIMO nonlinear systems with partial tracking errors constrained," IEEE Transactions on Fuzzy Systems, vol. 23, no. 4, pp. 729-742, 2015.

[24] D. P. Li and D. J. Li, "Adaptive neural tracking control for an uncertain state constrained robotic manipulator with timevarying delays," IEEE Transactions on Systems, Man, and Cybernetics: Systems, 2017.

[25] B. Niu, X. Zhao, X. Fan, and Y. Cheng, "A new control method for state-constrained nonlinear switched systems with application to chemical process," International Journal of Control, vol. 88, no. 9, pp. 1693-1701, 2015.

[26] M. U. Khan, S. Li, Q. Wang, and Z. Shao, "CPS Oriented Control Design for Networked Surveillance Robots with Multiple Physical Constraints," IEEE Transactions on Computer-Aided Design of Integrated Circuits and Systems, vol. 35, no. 5, pp. 778-791, 2016.

[27] W. He and S. S. Ge, "Cooperative control of a nonuniform gantry crane with constrained tension," Automatica, vol. 66, pp. 146-154, 2016.

[28] K. P. Tee, S. S. Ge, and E. H. Tay, "Barrier Lyapunov Functions for the control of output-constrained nonlinear systems," Automatica, vol. 45, no. 4, pp. 918-927, 2009.

[29] Z. Liu, G. Lai, Y. Zhang, and C. L. Chen, "Adaptive neural output feedback control of output-constrained nonlinear systems with unknown output nonlinearity," IEEE Transactions on Neural Networks and Learning Systems, vol. 26, no. 8, pp. 1789-1802, 2015.

[30] H. Li, L. Bai, L. Wang, Q. Zhou, and H. Wang, "Adaptive neural control of uncertain nonstrict-feedback stochastic nonlinear systems with output constraint and unknown dead zone," IEEE Transactions on Systems, Man, and Cybernetics: Systems, vol. 47, no. 8, pp. 2048-2059, 2017.
[31] Y.-J. Liu and S. Tong, "Barrier Lyapunov functions for Nussbaum gain adaptive control of full state constrained nonlinear systems," Automatica, vol. 76, pp. 143-152, 2017.

[32] D. P. Li and D. J. Li, "Adaptive neural tracking control for nonlinear time-delay systems with full state constraints," IEEE Transactions on Systems, Man, and Cybernetics: Systems, vol. 47, no. 7, pp. 1590-1601, 2017.

[33] Y.-J. Liu, S. C. Tong, C. L. P. Chen, and D.-J. Li, "Adaptive $\mathrm{NN}$ control using integral barrier lyapunov functionals for uncertain nonlinear block-triangular constraint systems," IEEE Transactions on Cybernetics, vol. 47, no. 11, pp. 3747-3757, 2017.

[34] X. Jin, "Adaptive fault tolerant control for a class of input and state constrained MIMO nonlinear systems," International Journal of Robust and Nonlinear Control, vol. 26, no. 2, pp. 286302, 2016.

[35] K. P. Tee, B. Ren, and S. S. Ge, "Control of nonlinear systems with time-varying output constraints," Automatica, vol. 47, no. 11, pp. 2511-2516, 2011.

[36] Y. J. Liu, S. M. Lu, D. J. Li, and S. C. Tong, "Adaptive controller design-based ABLF for a class of nonlinear time-varying state constraint systems," IEEE Transactions on Systems, Man, and Cybernetics: Systems, vol. 47, no. 7, pp. 1546-1553, 2017.

[37] C.-W. Hung, C.-T. Lin, C.-W. Liu, and J.-Y. Yen, "A variablesampling controller for brushless DC motor drives with lowresolution position sensors," IEEE Transactions on Industrial Electronics, vol. 54, no. 5, pp. 2846-2852, 2007.

[38] J. Yao, Z. Jiao, and D. Ma, "Adaptive robust control of dc motors with extended state observer," IEEE Transactions on Industrial Electronics, vol. 61, no. 7, pp. 3630-3637, 2014.

[39] G. Yang, J. Yao, G. Le, and D. Ma, "Adaptive robust control of DC motors with time-varying output constraints," in Proceedings of the 34th Chinese Control Conference (CCC '15), pp. 4256-4261, China, July 2015.

[40] L. Ma, S. Lu, M. Gong, J. Chen, and Y. Liu, "Time-varying output constraint for DC motor control," in Proceedings of the $3 r d$ International Conference on Informative and Cybernetics for Computational Social Systems (ICCSS '16), pp. 151-154, China, August 2016. 


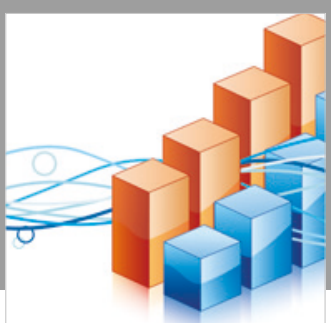

Advances in

Operations Research

\section{-n-m}
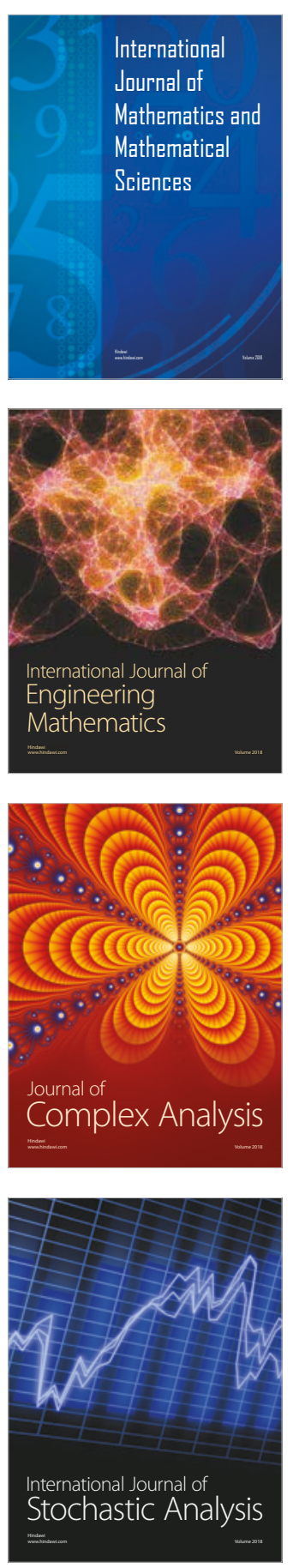
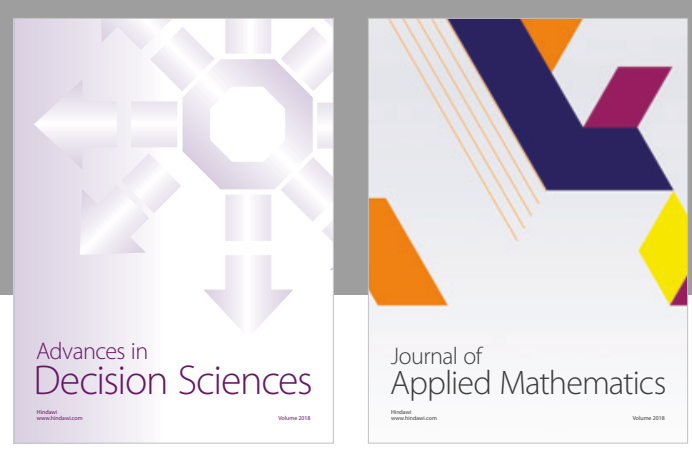

Journal of

Applied Mathematics
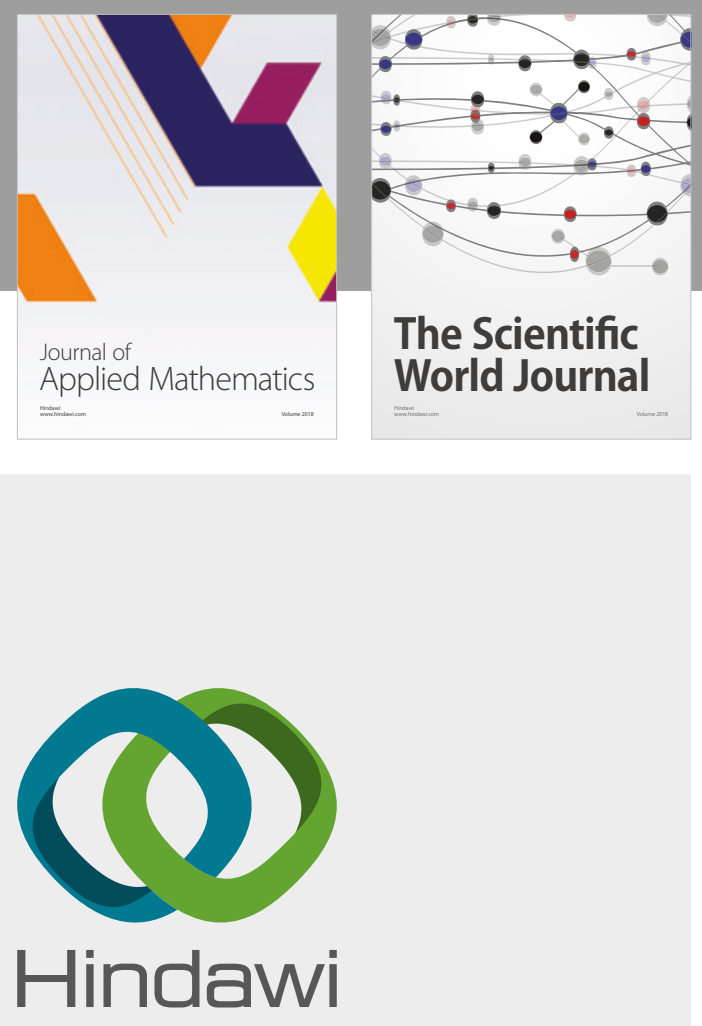

Submit your manuscripts at

www.hindawi.com

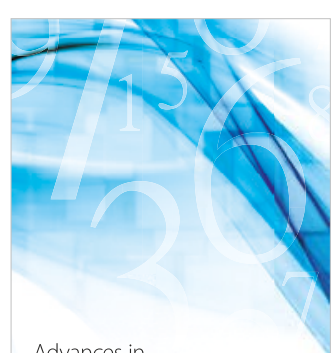

Advances in
Numerical Analysis
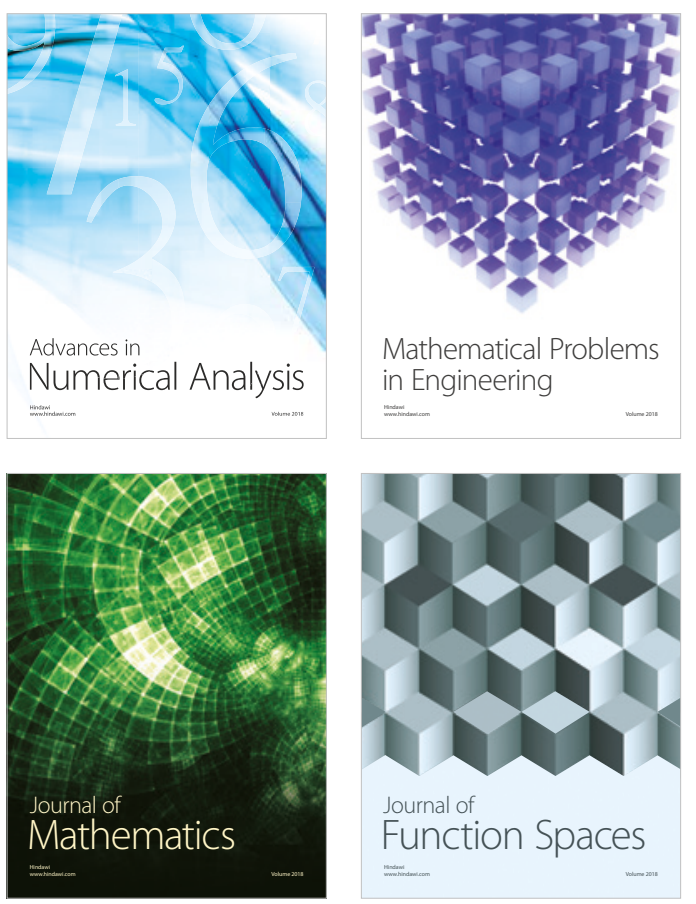

Mathematical Problems in Engineering

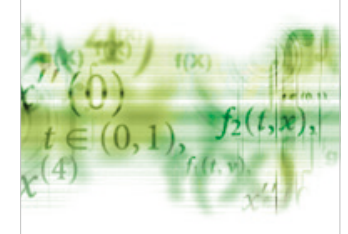

International Journal of

Differential Equations

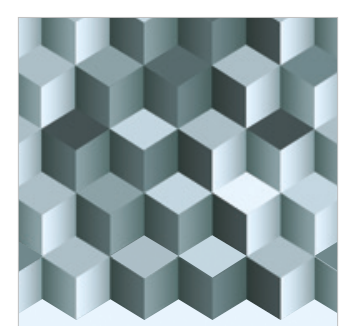

Journal of

Function Spaces
The Scientific

World Journal

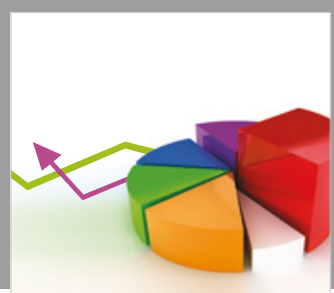

Journal of

Probability and Statistics
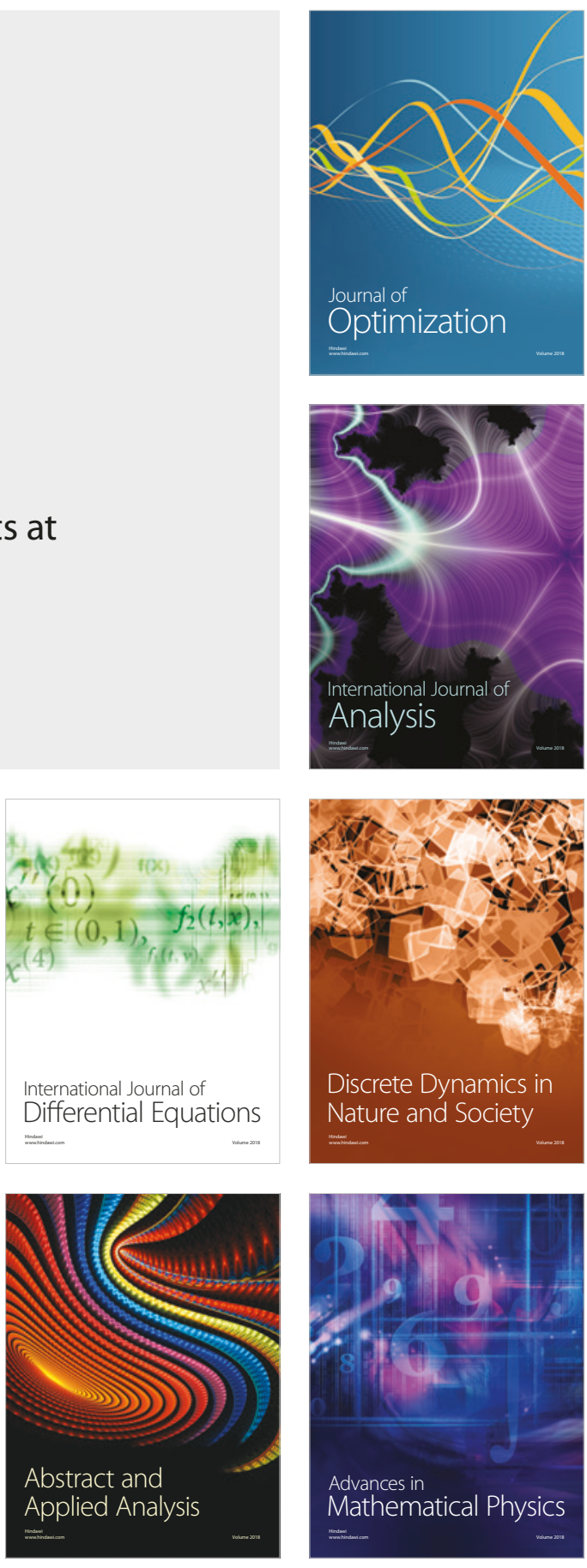